\title{
On Fully Closed Stable Modules
}

\author{
Shukur AL-AEASHI ${ }^{1, *}$ and Bijan DAVVAZ ${ }^{2}$ \\ ${ }^{1}$ Department of Mathematics, College of Education, University of Kufa, Iraq \\ ${ }^{2}$ Department of Mathematics, Yazd University, Yazd, Iran
}

("Corresponding author's e-mail: shukur.mobred@uokufa.edu.iq)

Received: 18 February 2020, Revised: 14 October 2020, Accepted: 10 November 2020

\begin{abstract}
In this paper, we studied the notion of the fully closed stable module and identified some basic properties of this notion. We also investigated some concepts which are related to this module. In addition, the notion of CL-duo and fully closed stable modules were also studied.
\end{abstract}

Keywords: CL-duo module, Fully stable module, Fully closed stable module, Mono-C-coretractable module

\section{Introduction}

All rings are associative with identity, and modules are unitary. Let $\mathrm{M}$ be an R-module. It should be recalled that a submodule $N$ of $M$ is called stable (fully invariant), if $N$ contains $f(N)$ for each $R$ homomorphism $\mathrm{f}: \mathrm{N} \rightarrow \mathrm{M}$ ( $\mathrm{f}: \mathrm{M} \rightarrow \mathrm{M}$ ). Likewise, an R-module $\mathrm{M}$ is called fully stable (duo) if each submodule of $\mathrm{M}$ is stable (fully invariant), [1,7]. It is clear that every fully stable module is duo but the converse may not be true. A submodule $\mathrm{N}$ of an $\mathrm{R}$-module $\mathrm{M}$ is said to be closed in $\mathrm{M}$ (denoted by $\mathrm{N} \leq \mathrm{c}$ $M$ ), if $N$ has no proper essential extensions in $M$, that means if $N \leq e K \leq M$, then $N=K$. Every direct summand of any R-module $\mathrm{M}$ is a closed submodule in $\mathrm{M}$. Recall that a module $\mathrm{M}$ is called Rickart if the annihilator in $M$ of any single element of $S=\operatorname{End}(M)$ is generated by an idempotent of $S$ and $M$ is called a dual Rickart module if the image in $\mathrm{M}$ of any single element of $\mathrm{S}$ is generated by an idempotent of $\mathrm{S}$ [10]. Recall that a module $M$ is said to be dual strongly Rickart if the image of any single element of $\mathrm{S}=\mathrm{End}(\mathrm{M})$ is generated by a semicentral idempotent element of S. [4].

Definition(1) [1] An R-module $M$ is called fully closed stable (Briefly, fully c-stable) if every closed submodule is stable. A ring $\mathrm{R}$ is fully c-stable ring if $\mathrm{R}$ is fully c-stable $\mathrm{R}$-module.

\section{Examples and remarks(2):}

(1) It is clear that every fully stable is fully c-stable but not conversely.

(2) Z as Z-module is Fully c-stable but it is not fully stable.

(3) Recall that, a submodule $\mathrm{N}$ of $\mathrm{M}$ is said to satisfy Baer's criterion if for each $\beta: N \rightarrow M$ there exists an element $r \in R$ such hat $\beta(n)=r n$ for each $n \in N$. Thus we have the following:

Every module satisfies Baer condition i fit is fully stable and hence fully c-stable module.

(4) A simple closed module is fully c-stable. Where "A module $\mathrm{M}$ is called simple closed if $\mathrm{M}$ has only 2 closed submodules, which are 0 and M" [7].

(5) Recall that "A module $\mathrm{M}$ is mono-C-coretractable module if for each proper closed submodule $\mathrm{N}$ of $\mathrm{M}$, there exists a monomorphism $\mathrm{f}$ from $\mathrm{M} / \mathrm{N}$ into $\mathrm{M}$ " [8]

Every mono-C-coretractable module implies fully c-stable. 
(6) Recall that, a ring $\mathrm{R}$ is called Quasi-Frobenius, satisfying that $\mathrm{R}$ as a left $\mathrm{R}$-module is Noetherian. It is mentioned in [1] that every Quasi-Frobenius ring is fully stable, and hence fully c-stable.

(7) Let $M \cong M^{\prime}$ and $M$ is fully c-stable R-module, then $M^{\prime}$ is also fully c-stable.

(8) If $\mathrm{R}$ is a fully c-stable ring and $\mathrm{M}$ be a faithful cyclic R-module. Then $\mathrm{M}$ is fully c-stable.

Proof: Let $M=a R$ for some $a, E$. Then $R \cong a R$ since $M$ is a faithful cyclic module. But $R$ is fully c-stable. So $\mathrm{M}$ is fully c-stable by part (7).

\section{Fully closed stable module}

Proposition(1): [1, lemma 2.8]An R-module $\mathrm{M}$ is fully c-stable if and only if every submodule of $\mathrm{M}$ is essential in a stable submodule of $\mathrm{M}$.

Proposition(2): Let $\mathrm{M}$ be a fully c-stable module and $\mathrm{N}$ be a closed submodule of $\mathrm{M}$, then $\mathrm{N}$ is fully cstable too.

Proof: Let $\mathrm{K}$ be a closed submodule in $\mathrm{N}$ and $\mathrm{f}: \mathrm{K} \rightarrow \mathrm{N}$ be an $\mathrm{R}$-homomorphism, we have inclusion map $\mathrm{i}: \mathrm{N} \rightarrow \mathrm{M}$ and $\mathrm{K}$ is also closed in $\mathrm{M}$, so by fully c-stability $\mathrm{i}^{\circ} \mathrm{f}(\mathrm{K}) \subseteq \mathrm{K}$ implies $\mathrm{f}(\mathrm{K}) \subseteq \mathrm{K}$. Thus $\mathrm{N}$ is fully cstable module.

Proposition(3): Let $\mathrm{N}$ be a direct summand of a fully c-stable R-module $\mathrm{M}$. Then $\mathrm{N}$ is fully c-stable.

Proof: Let $\mathrm{N}$ be a direct summand of $\mathrm{M}$. Then $\mathrm{M}=\mathrm{N} \oplus \mathrm{W}$ for some submodule $\mathrm{W}$ of $\mathrm{M}$. Let $\mathrm{K}$ be a proper closed submodule of $\mathrm{N}$ which implies that $\mathrm{N}$ is a proper closed submodule of $\mathrm{M}$. But $\mathrm{M}$ is fully c-stable, so $\mathrm{K}$ is fully c-stable of $\mathrm{M}$ and hence $\mathrm{K}$ is stable in $\mathrm{N}$. Thus $\mathrm{N}$ is a fully c-stable module. $\square$

Proposition(4): Let $\mathrm{M}$ be a mono-C-coretractable and fully c-stable R-module. Then every nonzero closed submodule of $\mathrm{M}$ is also mono-C-coretractable module.

Proof: Suppose that $\mathrm{N}$ is a nonzero closed submodule of $\mathrm{M}$. Let $\mathrm{K}$ be a proper closed submodule of N, so $\mathrm{K}$ is a closed submodule of $\mathrm{M}$. Since $\mathrm{M}$ is a mono-C-coretractable module, it follows that there exists $\mathrm{f} \in \operatorname{End}_{\mathrm{R}}(\mathrm{M}), \mathrm{f} \neq 0$ and $\mathrm{K}=\mathrm{kerf}$, so if $\mathrm{f}(\mathrm{N})=0$, then $\mathrm{N} \subseteq \mathrm{kerf}=\mathrm{K}$ so $\mathrm{N}=\mathrm{K}$ which is a contradiction. Thus $\mathrm{f}(\mathrm{N}) \neq 0$. Let $\mathrm{g}$ be the restriction map off on $\mathrm{N}$. Since $M$ is fully c-stable, so $g(N) \subseteq N$. Hence $g \in$ End $_{R}(N)$ and $\mathrm{g} \neq 0$ since $\mathrm{g}(\mathrm{N})=\mathrm{f}(\mathrm{N}) \neq 0$. Moreover $\mathrm{g}(\mathrm{K})=\mathrm{f}(\mathrm{K})=0$ and so $\mathrm{K} \subseteq$ kerg $\subseteq$ kerf $=\mathrm{K}$. Thus $\mathrm{K}=\mathrm{kerg}$ and hence $\mathrm{N}$ is mono-C-coretractable. $\square$

Proposition(5): Let $\mathrm{M}$ be a semisimple module. Then $\mathrm{M}$ is fully c-stable if and only if $\mathrm{M}$ is fully stable.

Proof: Since $\mathrm{M}$ is semisimple and hence every submodules of $\mathrm{M}$ a direct summand and hence it is closed in $M$. Then $M$ is fully c-stable if and only if $M$ is fully stable.

Consider $\mathrm{M}=\mathrm{Z}_{2} \oplus \mathrm{Z}_{2}$ as $\mathrm{Z}$-module. Then $\mathrm{M}$ is semisimple but not fully stable ( see [13]). Thus $\mathrm{M}$ is not fully c-stable.

Before the following proposition, we shall introduce the concept of closed injective Rmonomorphism. An R-monomorphism $\mathrm{f}: \mathrm{M} \rightarrow \mathrm{M}^{*}$ is called closed injective if Imf is closed submodule of $\mathrm{M}^{*}[2]$.

Proposition(6): Let $\mathrm{M}$ be an R-module. Then $\mathrm{M}^{*}$ is a fully c-stable if and only if Imf $\subseteq$ Img for each 2 homomorphisms $\mathrm{f}, \mathrm{g}: \mathrm{M} \rightarrow \mathrm{M}^{*}$ with $\mathrm{g}$ is closed injective.

Proof: $(\Rightarrow)$ Let $f, g: M \rightarrow M^{*}$ with $g$ is closed injective. Let $N=I m g$. Then there exists $h: N \rightarrow M$ such that $\mathrm{h} \circ \mathrm{f}=1_{\mathrm{M}}$ let $\beta=\mathrm{f} \circ \mathrm{h}: \mathrm{N} \rightarrow \mathrm{M}^{*}$, but $\mathrm{N}$ is closed stable in $\mathrm{M}^{*}$, so we have $\beta(\mathrm{N}) \subseteq \mathrm{N}$ which implies $\mathrm{f}(\mathrm{h}(\mathrm{N})) \subseteq$ $\mathrm{N}$, that is, $\operatorname{Im} \mathrm{f} \subseteq \operatorname{Img}$.

$(\Leftarrow)$ If $\mathrm{N}$ is a closed submodule of $\mathrm{M}^{*}$, and $\mathrm{f}: \mathrm{N} \rightarrow \mathrm{M}^{*}$ be a homomorphism, let $\mathrm{i}: \mathrm{N} \rightarrow \mathrm{M}^{*}$ be the inclusion map (which is closed injective) then by hypothesis $\mathrm{f}(\mathrm{N}) \subseteq \mathrm{i}(\mathrm{N}) \subseteq \mathrm{N}$, hence $\mathrm{N}$ is closed stable. Thus $\mathrm{M}^{*}$ is fully c-stable module. $\square$

Next, we have some corollaries about this proposition: 
http://wjst.wu.ac.th

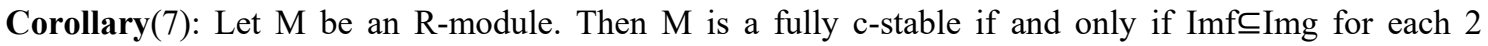
homomorphisms $f, g \in \operatorname{End}(M)$, with $g$ is closed injective.

Corollary(8): Let $\mathrm{M}$ be a dual Rickart R-module. Then $\mathrm{M}$ is a fully c-stable if and only if $\operatorname{Imf} \subseteq \operatorname{Img}$ for each 2 homomorphisms $\mathrm{f}, \mathrm{g} \in \operatorname{End}(\mathrm{M})$, with $\mathrm{g}$ is injective.

Corollary(9): Let $\mathrm{M}$ be a dual strongly Rickart R-module. Then $\mathrm{M}$ is a fully c-stable if and only if ImfᄃImg for each 2 homomorphisms f, $\mathrm{g} \in \operatorname{End}(\mathrm{M})$.

Proposition(10): Let $\mathrm{M}$ be an R-module. Then the following statements are equivalent:

(i) $\quad \mathrm{M}$ is fully c-stable module;

(ii) Every closed submodule of $\mathrm{M}$ is stable;

(iii) If $\mathrm{N}$ and $\mathrm{K}$ are 2 submodules of $\mathrm{M}$ such that $\mathrm{K}$ is closed in $\mathrm{M}$ and $\mathrm{N}$ is epimorphism image of $\mathrm{K}$ then $\mathrm{N} \subseteq \mathrm{K}$.

Proof: (i) $\Leftrightarrow$ (ii) It is clear.

(ii) $\Rightarrow$ (iii) Let $\mathrm{N} \leq \mathrm{M}$ and $\mathrm{K}$ be a closed submodule of $\mathrm{M}$ and $\mathrm{f}: \mathrm{K} \rightarrow \mathrm{N}$ be an epimorphism. Since $\mathrm{K}$ is closed in $M$ putting $i \circ f: K \rightarrow M$ where $i$ is the inclusion map from $N$ into $M, i \circ f(K) \subseteq K$ since $K$ is stable by hypothesis, then $\mathrm{f}(\mathrm{K}) \subseteq \mathrm{K}$, but $\mathrm{f}(\mathrm{K})=\mathrm{N}$ thus $\mathrm{N} \subseteq \mathrm{K}$.

(iii) $\Rightarrow$ (i) Let $\mathrm{N}$ be a closed submodule of $\mathrm{M}$ and $\mathrm{f}: \mathrm{N} \rightarrow \mathrm{M}$ be a homomorphism, then $\mathrm{f}: \mathrm{N} \rightarrow \mathrm{f}(\mathrm{N})$ is epimorphism by putting $\mathrm{f}(\mathrm{N})=\mathrm{K}$, so $\mathrm{K}=\mathrm{f}(\mathrm{N}) \subseteq \mathrm{N}$. Thus $\mathrm{M}$ is fully c-stable module. $\square$

A fully c-stable R-module $\mathrm{M}$ can be characterized using the trace of a closed submodule $\mathrm{N}$ of $\mathrm{M}$. Where $\mathrm{M}$ is fully c-stable if and only if every closed submodule $\mathrm{N}$ of $\mathrm{M}$ is equal to its trace in $\mathrm{M}$, where the trace of a submodule $N$ of $M$ in $M$ is the $\operatorname{set} \operatorname{tr}(N, M)=\left\{\sum_{i=1}^{k} f_{i}\left(n_{i}\right): f_{i} \in \operatorname{Hom}(N, M), n_{i} \in N, k \in \mathbb{N}\right\}$ [14].

Proposition(11): If $\mathrm{M}$ is fully c-stable R-module, then every closed monomorphism in End(M) is epimorphism.

Proof: Let $f \in \operatorname{End}(M)$ be a closed monomorphism that is $f(M)$ is closed in $M$. Define $g: f(M) \rightarrow M$ by $\mathrm{g}(\mathrm{f}(\mathrm{m}))=\mathrm{m}$ for all $\mathrm{m} \in \mathrm{M}, \mathrm{g}$ is well-defined and epimorphism but $\mathrm{M}$ is fully c-stable and $\mathrm{f}(\mathrm{M})$ is closed in $M$, so $g(f(M)) \subseteq f(M)$ but $g(f(M))=M$ since $g$ is epimorphism that is $M \subseteq f(M)$ hence $M=f(M)$. Therefore, $f$ is epimorphism. $\square$

Recall that " An R-module M is called c-Rickart if kerf is closed submodule of $\mathrm{M}$ for all $\mathrm{f} \in$ End(M) " [7].

Proposition(12): Let $\mathrm{M}$ be a c-Rickart R-module. If $\mathrm{M}$ is fully c-stable, then every monomorphism in End(M) is an epimorphism.

Proof: Since every monomorphism in End(M) of c-Rickart module is closed monomorphism, it follows the result.

Recall that " An R-module $M$ is called cohopfian if every R-monomorphism $\mathrm{f:} M \rightarrow \mathrm{M}$ is an isomorphism " [6]. Note that every Artinian R-module is cohopfian.

Corollary(13): Let $\mathrm{M}$ be a c-Rickart R-module. If $\mathrm{M}$ is fully c-stable, then $\mathrm{m}$ is cohopfian.

According to these results, we can see that every fully c-stable can be embedded in any closed submodule of $M$. Since let $f: M \rightarrow N$ is monomorphism where $N$ be a closed submodule of $M$, then foi: $\mathrm{M} \rightarrow \mathrm{M}$ is monomorphism in EndM by above $\mathrm{f} \circ \mathrm{i}$ is isomorphism since $\mathrm{M}$ is fully c-stable that is $\mathrm{f} \circ \mathrm{i}(\mathrm{M})=\mathrm{f}(\mathrm{i}(\mathrm{M}))=\mathrm{f}(\mathrm{M}) \subseteq \mathrm{N}$.

Proposition(14): Let $M$ be an R-module. Then $M$ is fully c-stable module if and only if $M$ is satisfied Baer 's criterion on each closed cyclic submodule $\mathrm{N}$ of $\mathrm{M}$. 
http://wjst.wu.ac.th

Proof: $(\Rightarrow)$ Let $N=R_{x}$ be a cyclic closed in $M$ and $f: N \rightarrow M$ be an homomorphism. Since $M$ is fully cstable so $f(N) \subseteq N$ that is $f\left(R_{x}\right) \subseteq R_{X}$, for each $x \in N=R_{x}$ there exists $r \in R$ such that $x=r x$ that is $M$ satisfy Baer's criterion.

$(\Leftarrow)$ It is clear by $\operatorname{Remark}(1.2(3))$. $\square$

\section{CL-duo model}

In this section, we shall study the notion of fully c-stable module with a generalization of duo module. First, we recall duo property of modules relative to closed submodules. Where an R-module M is called CL-duo if for each closed submodule $\mathrm{N}$ of $\mathrm{M}$ is fully invariant. A ring $\mathrm{R}$ is CL-duo if $\mathrm{R}$ is CL-duo R-module which is defined in [3].

Definition(1): An R-module $\mathrm{M}$ is called CL-duo if for each closed submodule $\mathrm{N}$ of $\mathrm{M}$ is fully invariant. A ring $\mathrm{R}$ is $\mathrm{CL}$-duo if $\mathrm{R}$ is $\mathrm{CL}$-duo $\mathrm{R}$-module.

Now, we shall recall some remarks about CL-duo:

\section{Remarks and Examples(2):}

(1) Every duo R-module is CL-duo.

(2) Every fully c-stable R-module is CL-duo.

(3) The multiplication module is duo and hence CL-duo.

(4) Every CL-duo is weak duo, where a module M is weak duo if each direct summand in M is fully invariant.

(5) One can see a module M is CL-duo if and only if each closed cyclic submodule is fully invariant.

(6) Recall that an $\mathrm{R}$-module $\mathrm{M}$ is quasi-projective if for each submodule $\mathrm{N}$ of $\mathrm{M}$ and each $\mathrm{R}$ homomorphism $\mathrm{f}: \mathrm{M} \rightarrow \mathrm{M} / \mathrm{N}$, there exists an R-endomorphism $\mathrm{g}$ of $\mathrm{M}$ such that $\mathrm{f}=\pi \circ \mathrm{g}$ where $\pi: \mathrm{M} \rightarrow \mathrm{M} / \mathrm{N}$ is the natural epimorphism[6].Clearly, under the class quasi-projective the 2 concepts fully c-stable and CL-duo are coincide.

Proposition(3): Let $\mathrm{M}=\mathrm{M}_{1} \oplus \mathrm{M}_{2}$ be a CL-duo R-module then $\mathrm{M}_{1}$ and $\mathrm{M}_{2}$ are also CL-duo.

Proof: see [3, proposition 1.3.]

In general, a closed submodule of CL-duo needs not be a CL-duo module. However, if under skewinjective is true, where a module $\mathrm{M}$ is skew-injective, each endomorphism of each submodule can be extended to an endomorphism of M[12]. Also, we have the following proposition.

Proposition(4): Let $\mathrm{M}$ be a CL-duo R-module. If $\mathrm{M}$ is skew-injective, then every closed submodule of $\mathrm{M}$ is CL-duo.

Proof: Let $K$ be a closed submodule of $M$ and $N$ be a closed submodule of $K, f: K \rightarrow K$ since $M$ is skewinjective so $f$ can be extended to $\bar{f} \in \operatorname{End}(M)$ hence $f(N)=\bar{f}(N) \subseteq N$ since $M$ is CL-duo module. Thus $K$ is also CL-duo. $\square$

We can generalize this concept by taking only the closed submodules as an $\mathrm{R}$-module. $\mathrm{M}$ is closed quasi-projective if for each closed submodule $\mathrm{N}$ of $\mathrm{M}$ and each $\mathrm{R}$-homomorphism $\mathrm{f}: \mathrm{M} \rightarrow \mathrm{M} / \mathrm{N}$, there exists an R-endomorphism $g$ of $\mathrm{M}$ such that $\mathrm{f}=\pi \circ \mathrm{g}$ where $\pi: \mathrm{M} \rightarrow \mathrm{M} / \mathrm{N}$ is the natural epimorphism. Clear that the following diagram is satisfied;

$$
\text { projective } \Rightarrow \text { quasi-projective } \Rightarrow \text { closed quasi-projective }
$$


http://wjst.wu.ac.th

We shall call an R-module $\mathrm{M}$ as closed-multiplication (shortly, c-multiplication) if for each closed submodule $\mathrm{N}$ of $\mathrm{M}$ is equal to IM for some ideal I of $\mathrm{R}$. This concept is in fact a generalization of multiplication modules[9], and hence every multiplication module is c-multiplication. Moreover, it is known that every multiplication module is duo module, so we can generalize this by the following:

Proposition(5): Every c-multiplication R-module is CL-duo.

Proof: Let $N$ be a closed submodule of $M, N=I M$ for some $I \leq R, f: M \rightarrow M$. $f(N)=f(I M)=I f(M) \subseteq I M=N$. Thus $\mathrm{M}$ is CL-duo.

Proposition(6): Let $\mathrm{M}$ be a $\mathrm{R}$-module. If $\mathrm{M}$ is semisimple. Then the following are equivalent:
(i) $\mathrm{M}$ is duo,
(ii) $\mathrm{M}$ is fully c-stable,
(iii) $\mathrm{M}$ is fully stable,
(iv) $\quad \mathrm{M}$ is CL-duo,
(v) $\mathrm{M}$ is weak duo

\section{Proof:}

(i) $\Rightarrow$ (ii) It is straightforward.

(ii) $\Leftrightarrow$ (iii) By corollary 2.5, it is satisfied under the semisimple condition.

(iii) $\Leftrightarrow$ (iv) $\Leftrightarrow(\mathrm{v}) \Leftrightarrow$ (i) It is clear.

Proposition(7): Let $\mathrm{M}$ be a R-module. If $\mathrm{M}$ is simple closed, then the following are equivalent:

(i) $\quad \mathrm{M}$ is CL-duo;

(ii) $\quad \mathrm{M}$ is fully c-stable;

(iii) $\mathrm{M}$ is weak duo

Proof: Since $\mathrm{M}$ is simple closed, it follows that $\mathrm{M}$ has no proper closed submodule except 0 and $\mathrm{M}$. Therefore, we can easily prove this equivalent.

Proposition(8): Let $\mathrm{M}$ be a closed injective R-module. Then every closed fully invariant submodule $\mathrm{N}$ of $M$ is stable.

Proof. Let $\mathrm{N}$ be a closed submodule of $\mathrm{M} \mathrm{f,g}: \mathrm{N} \rightarrow \mathrm{M}$. Since $\mathrm{M}$ is closed injective, there exists $\mathrm{h}: \mathrm{M} \rightarrow$ $M$ such that $g \circ i=f$ but $N$ is fully invariant that is $g(N) \subseteq N$, but $f(N)=g \circ i(N)=g(N)$ then $f(N) \subseteq N$ which implies $\mathrm{N}$ is stable. $\square$

Proposition(9): Let $\mathrm{M}$ be a closed injective R-module. If $\mathrm{M}$ is CL-duo, then $\mathrm{M}$ is fully c-stable.

Proof. By Proposition 3.7 and remark 3(2).

\section{Conclusions}

Many new classes of the fully stable module have been discovered recently. All of these have attracted researchers in the field to investigate them in detail. This article studies the fully closed stable modules as a generalization of the fully stable modules. Results were presented following the study on fully closed stable modules. Furthermore, we investigated the notion of fully closed stable modules with some generalizations about the duo module.

\section{Acknowledgments}

The authors would like to thank the referees for reading the manuscript carefully and for giving their helpful comments. 


\section{References}

[1] MS Abbas and SA Al-Saadi. Stable (Quasi-) continuous modules. Al-Mustansiriya J. Sci. 2013; 24, 83-93.

[2] MS Abbas. Semi-injectivity and continuity. Al-Mustansiriya J. Sci. 2007; 18, 99-112.

[3] MA Ahmed. CL-duo modules. Baghdad Sci. J. 2017; 14, 642-50.

[4] SA Al-Saadi and TA Ibrahim. Dual strongly Rickart modules. J. Adv. Math. 2015; 11, 3923-30.

[5] ZA El-Bast and PF Smith. Multiplication modules. Comm. Algebra 1988; 16, 755-79.

[6] Y Gang and L Zhong-Kui. On hopfian and co-hopfian modules. Vietnam J. Math. 2007; 35, 73-80.

[7] IM Ali and TY Ghawi. On closed Rickart modules. Iraqi J. Sci. 2016; 57, 2746-53.

[8] IMA Hadi and SN Al-Aeashi. C-coretractable and strongly C-coretractable modules. In Proceedings of the $1^{\text {st }}$ Scientific International Conference, College Of Science, Al-Nahrain University. 2019, p. $65-72$.

[9] SN Al-Aeashi and IMA Hadi. Closede multiplication modules. Ital. J. Pure Appl. Math.; 2019, 46.

[10] G Lee. 2010, Theory of Rickart modules. Ph. D. Dissertation. Ohio State University, Ohio State USA.

[11] MS Abbas and AG Naum. Fully stable modules. Iraqi J. Sci. 2002; 43, 62-74.

[12] AC Ozcan, A Harmanci and PF Smith. Duo modules. Glasgow Math. J. 2006; 48, 533-45.

[13] A Tuganbaev. Skew-injective modules. J. Math. Sci. 1999; 95, 2328-420.

[14] R Wisbauer. Foundations of modules and ring theory. Gordon and Breach Science Publishers, Reading, 1991. 